\title{
Case Report of Systemic Lupus Erythematous
}

\author{
${ }^{1}$ Henry A Mayala, ${ }^{2}$ Levy Kwirisiima, ${ }^{3}$ Peter Kisenge, ${ }^{4}$ Smita, ${ }^{5}$ Rweyemamu, ${ }^{6}$ Kawajika, \\ ${ }^{7}$ Banduka, ${ }^{8}$ Mghanga Fabian, ${ }^{9}$ Patrick Mirumbe
}

\begin{abstract}
BACKGROUND: SLE is an autoimmune disease characterized by the production of unusual antibodies in the blood. The cause is unknown 1

CASE PRESENTATION: In this case report a 49 year old lady who is a known hypertensive on medication (losartan potassium 50mg od), admitted at the cardiovascular medicine department of $\mathrm{MNH}$ with the chief complains of: productive cough, difficulty in breathing, awareness of heart beat and episodes of tonic clonic seizures, on examination she had rashes on her trunk and extremities, clinical and laboratory finding are suggestive of SLE and it was confirmed according to American Rheumatology Association criteria's.
\end{abstract}

CONCLUSION: SLE is a rare autoimmune disorder in Africa black population, it is more common in Asia and Europe, but we shoulkd be very alert that due to enviromental changes the disorder shoukd not be overlooked, and we need to understand its presentation so that it might not go unnoticed.

Keywords: SLE, DsDNA, ANA, ASOT,African, Where SLE- systemic lupus erythematosus, DsDNA- double strand deoxybonucleated acid, ANA- Antinuclear antibody,ASOT- Anti streptolysin O titre,CBC- complete blood count

\section{INTRODUCTION:}

SLE is an autoimmune disease characterized by the production of unusual antibodies in the blood, SLE is much more common in women than in men. The cause is usually unknown. Lupus can cause disease of the skin, kidney, joints and or nervous system. When only the skin is involved, the condition is called lupus dermatitis or cutaneous lupus erythematosus. A form of lupus dermatitis that can be isolated to the skin without internal disease is called discoid lupus. when internal organs are involved, the condition is reffered to as SLE. The disease can affect all ages but most commonly begins from 20-45yrs of age. the patient with SLE presents with fatigue, low grade fever, muscle aches, hair loss, arthritis, poor circulation to the fingers and toes with cold exposure, facial rash. If the lungs are involved the patient will present with difficulty in breathing and productive cough sometimes mixed with blood.

11 criteria for diagnosing SLE according to American Rheumatism Association:

1. Malar butterfly rash

2. Discoid skin rash (patchy redness with hyperpimented and hypopigmented)

3. Photosensitivity

4. Mucus membrane ulcers

5. Arthritis (two or more swollen, tender joint of extremities)

6. Pleuritis or pericarditis

7. Kidney abnormalities

8. Brain irritation (manifested by seizures and or psychosis, lupus cerebritis)

9. low WBC or RBC or platelet count

10. Anti DNA

11. ANA

For one to diagnose SLE 4 or more criteria's should be present. 


\section{CASE PRESENTATION:}

We present a case report of a 49 years old female, a known hypertensive on losartan potassium 50mg od, admitted at cardiovascular medicine department of Muhimbili National Hospital with the chief complains of productive cough, difficulty in breathing, awareness of heart beat, on examination she is conscious, sparse silky hair with hair loss, residual hyperpigmented macules wide spread on the trunk and extremities, pale and she is dyspneic. on admission the following investigations were done: EKG, chest $x$-ray, Echocardiography, CBC, ANA, DsDNA, ASOT, Renal function test, Liver function test EKG revealed left ventricular hypertrophy, chest x-ray revealed cardiomegaly, echocardiography revealed LVH with borderline LV systolic dysfunction, grade II diastolic dysfunction,

CBC revealed low RBC count of $2.19 \mathrm{~m} / \mu \mathrm{l}$

Antinuclear antibody positive 1:640

Double strand DNA positive 1:20

ASOT negative

Renal function test and liver function test were normal

Thus our patient had discoid skin rash, brain irritation (seizures), low RBC count, positive double strand DNA and positive antinuclear antibody suggesting the diagnosis of SLE according to American Rheumatism Association.

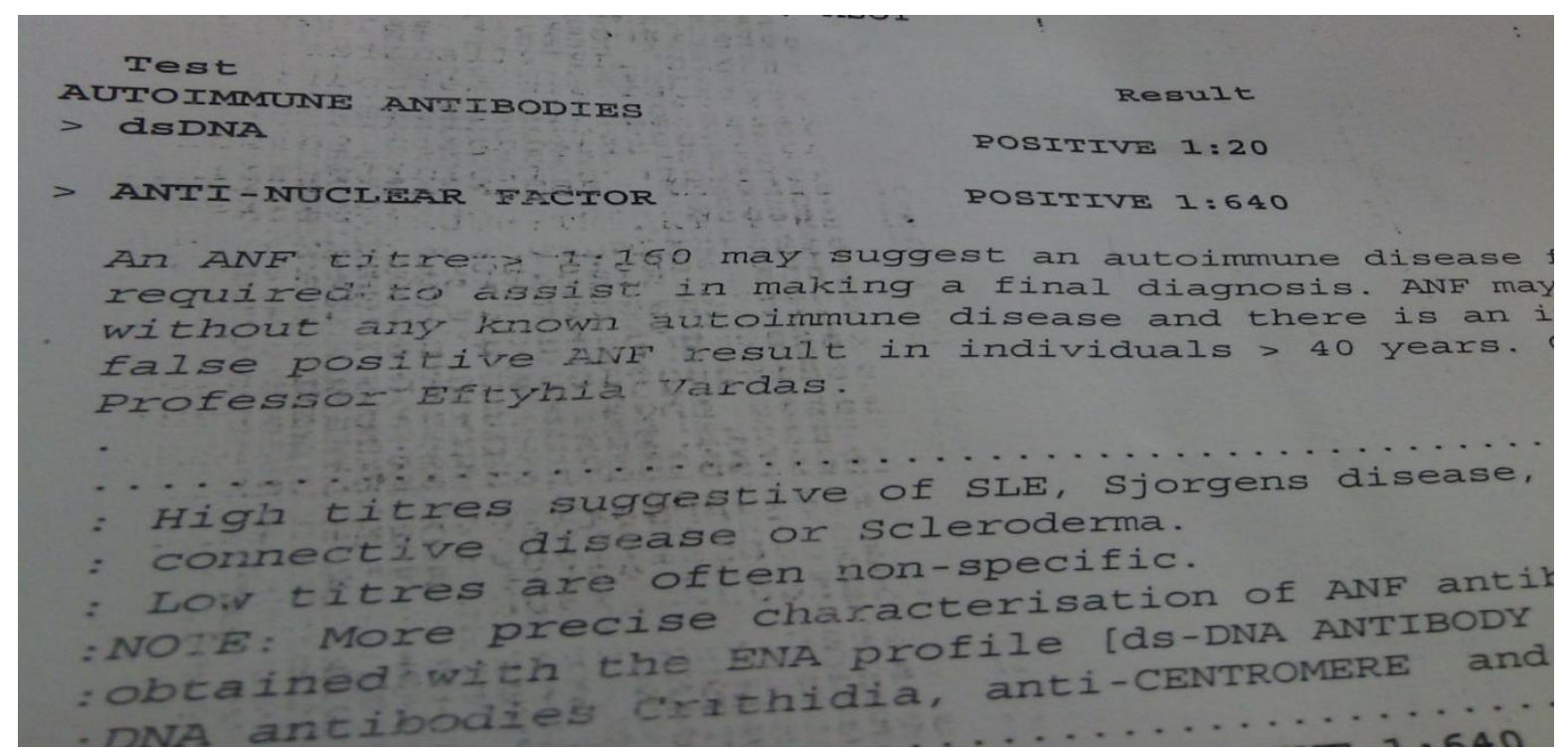

FIGURE: 1 showing the results of Double strand DNA and Antinuclear antibody



FIGURE: 2 ECHO report showing LV Systolic function findings 


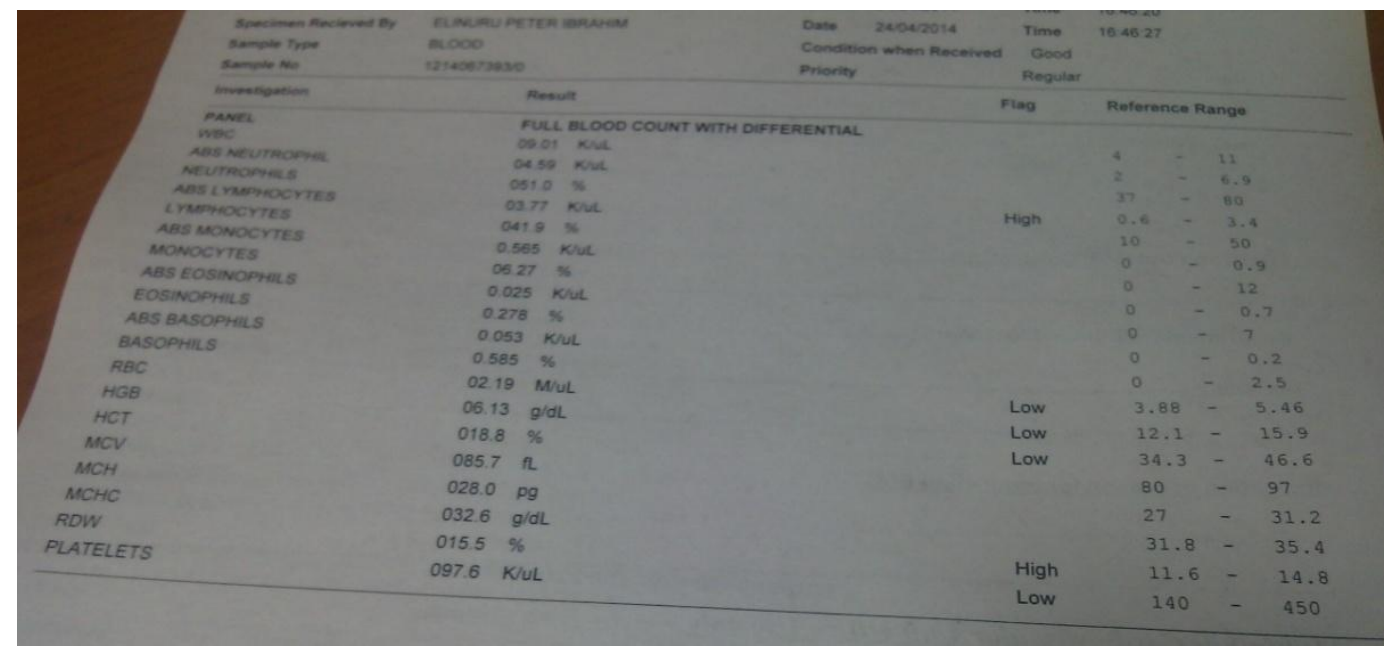

FIGURE: 3 CBC results showing low RBC count

\section{DISCUSSION:}

SLE prevalence is higher among Asians, Afro-Americans. Afro-Caribbean's and Hispanic American's compared to Americans of European descent in the USA, SLE occurs infrequent in blacks in Africa .I had to report this condition, because the disease is rarely reported in blacks in Africa, suggesting that there may be an environmental trigger as well as genetic basis for the disease.SLE carries a highly variable prognosis for individual patients, the natural history of SLE ranges from a relatively benign disease to rapidly progressive and even fatal disease. Thus the influence of race on prognosis has been widely debated. The LUMINA study group examined SLE in blacks, white and Hispanic patients in USA and reported both disease activity and poverty predicted higher mortality in racial and ethnic minorities. The most important thing in management of SLE is stressing the importance of adherence to medications and follow-up appointments, because of their risk for infection and cardiovascular disease. Currently our patient is on prednisolone $40 \mathrm{mg}$ od am, and $20 \mathrm{mg}$ od pm. Even though this is a rare disease it is of paramount importance to understand it, as it affects the black population. At this moment our patient has improved and has been discharged through internal medicine outpatient clinic.

\section{REFERENCES:}

[1]. Mayo clinic textbook of internal medicine $8^{\text {th }}$ edition

[2]. http://emedicine.medscape.com/article/332244

[3]. http://www.healthline.com/health/systemic-lupus-erythematosus

[4]. Crow MK. Etiology and pathogenesis of systemic lupus erythematosus. In: Firestein GS, Budd RC, Gabriel SE, et al, eds. Kelley's Textbook of Rheumatology. 9th ed. Philadelphia, Pa: Saunders Elsevier;2012:chap XX

[5]. http://www.nlm.nih.gov/medlineplus/ency/article/000435.htm

[6]. http://www.rheumatology.org/practice/clinical/patients/diseases_and_conditions/lupus.asp

[7]. http://www.nhs.uk/conditions/lupus/Pages/Introduction.aspx 